\title{
Germanica
}

\section{Amerikanismus und Neue Sachlichkeit in der deutschen Fotografie der zwanziger Jahre}

Américanisme et nouvelle objectivité dans l'art photographique des années vingt

Herbert Moiderings

\section{OpenEdition}

\section{Journals}

Édition électronique

URL : http://journals.openedition.org/germanica/2395

DOI : 10.4000/germanica.2395

ISSN : 2107-0784

Éditeur

Université de Lille

Édition imprimée

Date de publication : 31 décembre 1991

Pagination : 229-243

ISSN : 0984-2632

\section{Référence électronique}

Herbert Moiderings, « Amerikanismus und Neue Sachlichkeit in der deutschen Fotografie der zwanziger Jahre », Germanica [Online], 9 | 1991, Online erschienen am: 25 Februar 2014, abgerufen am 06 Oktober 2020. URL : http://journals.openedition.org/germanica/2395 ; DOI : https://doi.org/ 10.4000/germanica.2395

Ce document a été généré automatiquement le 6 octobre 2020.

(ㄷ) Tous droits réservés 


\title{
Amerikanismus und Neue Sachlichkeit in der deutschen Fotografie der zwanziger Jahre
}

Américanisme et nouvelle objectivité dans l'art photographique des années vingt

\author{
Herbert Moiderings
}

1 «Rußland oder Amerika? oder Rußland und Amerika?», lautete die Alternative, vor die der liberale Demokrat Harry Graf Kessler in einem Vortrag vom Juni 1919 die deutsche Politik nach der Novemberrevolution gestellt sah ${ }^{1}$.

2 Zehn Jahre später veröffentlichte der Architekt Erich Mendelsohn einen Bildband mit dem Titel «Rußland Europa Amerika» ${ }^{2}$, in dem er die neuesten architektonischen Leistungen in den drei Ländern und Kontinenten einer vergleichenden Betrachtung unterzog. Doch nicht die baukünstlerische Seite soll uns hier interessieren, sondern das historische Kräfteparallelogramm, das Mendelsohn in dem Buch entwirft. "Europa», schreibt er, «ist heute arm, krank von den Nachwirkungen des Krieges, voll wirtschaftlicher und politischer Gegensätze und Unsicherheiten»³. In Rußland sieht Mendelsohn den Beginn einer neuen sozialen Ordnung, «die Vehemenz des Gefühls» und des Kollektivs ${ }^{4}$, ein Experiment, dem er Interesse entgegenbringt, das ihn aber nicht ganz zu überzeugen vermag. Seine Liebe gilt Amerika, der Verkörperung des Individualismus und der «rationellen Klugheit» ${ }^{5}$. Mehr noch als diese geistigen Eigenschaften fesselt ihn jedoch der materielle Reichtum der Vereinigten Staaten: die gewaltige Überlegenheit ihrer Produktivkräfte. "Amerika ist Herr der Welt» ${ }^{6}$. Mit dieser Feststellung beschließt Mendelsohn seine kurze Charakterisierung der wirtschaftlichen und politischen Lage von 1929.

3 Amerika, erläutert er, das heißt planvolle Einstellung der Technik in den Dienst des Menschen als Grundlage der Erschaffung eines neues Lebens. Er fordert den Leser auf, «die Geistlosigkeit unseres egoistischen Gefühls (gemeint ist der Eurozentrismus, Anm. d. Verf.) einzutauschen gegen den umfassenden technischen Geist der neu werdenden Welt» ${ }^{7}$. 
Mendelsohns Ausführungen in «Rußland Europa Amerika» skizzieren beispielhaft die auseinanderstrebenden gesellschaftlichen und geistigen Tendenzen, zwischen denen die künstlerische Intelligenz in Deutschland nach dem 1. Weltkrieg zu wählen hatte. Für das ökonomisch und sozial zerrüttete Europa sieht er keine eigenständige Entwicklungsmöglichkeit mehr; es befindet sich in einer Sackgasse, eingezwängt zwischen den welthistorisch bestimmenden Kräften: dem mächtigsten und fortgeschrittensten Kapitalismus, den USA, und der sozialistischen Revolution, die 1917 in Rußland ihren Anfang genommen hatte.

5 Mendelsohns Thesen waren Bestandteil einer geistigen Strömung in der Weimarer Republik, die man «Amerikanismus» nannte. Um Bedeutung und Tragweite dieser Bewegung zu umreißen, sei ein kurzer Rückblick auf die wirtschaftlichen und politischen Verhältnisse in Deutschland nach 1918 vorausgeschickt.

6 Nachdem die Novemberrevolution durch die vereinten Kräfte der Sozialdemokratie und der Reaktion im Rahmen der bestehenden Eigentumsverhältnisse gehalten worden war, kam es 1923 zu einer erneuten revolutionären Krise. Die Nachkriegsinflation hatte $\mathrm{zu}$ einer totalen Geldentwertung geführt. Der Staat war zahlungsunfähig. Die französische Regierung nahm ausstehende Reparationszahlungen zum Vor wand, um das Ruhrgebiet $\mathrm{zu}$ besetzen, worauf die Arbeiterklasse geschlossen mit passivem Widerstand reagierte. Als die Reichswehr die gewählte SPD/KPDRegierung in SachsenThüringen mit Waffengewalt aus dem Amt trieb, gab die KPD-Führung die Losung des bewaffneten Aufstandes aus, schreckte jedoch im letzten Augenblick vor seiner Durchführung zurück. In Hamburg, wo die Rückzugsmeldung nicht mehr rechtzeitig eintraf, kam es zum Aufstand, der blutig niedergeschlagen wurde.

7 Diese Ereignisse hatten den Siegern des 1 . Weltkriegs, der englischen, französischen und vor allem der amerikanischen Bourgeoisie die Unmittelbarkeit der Gefahr einer sozialistischen Revolution vor Augen geführt. Eine solche Revolution drohte das wirtschaftliche und technische Potential Deutschlands mit dem der Sowjetunion zu vereinigen, und gegenüber dieser verbundenen Macht, das war allen Beteiligten klar, würde Europa bei einer erneuten revolutionären Welle nicht mehr standhalten können.

8 Nach 1918 wurden die USA zur Regierungszentrale der westlichen Welt. Sie setzten durch, daß unter Führung von General Dawes ein Sachverständigenausschuß gebildet wurde, dessen Aufgabe es war, Wege zu finden, um die Wirtschaft und die finanzielle Leistungsfähigkeit des deutschen Kapitalismus wiederherzustellen. Der «Dawes-Plan» war die Grundlage des Londoner Abkommens von 1924, dessen Verwirklichung Amerika den entscheidenden Einfluß auf die ökonomische und politische Entwicklung der Weimarer Republik garantierte. Ein spezielles Komitee hatte die Höhe der jeweiligen Reparationszahlungen so festzusetzen, daß die deutsche Währung nicht zu sehr destabilisiert wurde. Die Gewährung eines internationalen Kredits von 800 Millionen Gold-mark, dessen Hauptanteil die USA bereitstellten, führte zu einer vorübergehenden Belebung der deutschen Wirtschaft. Diesem "politischen Kredit» folgte ein immenser Export von Kapital seitens der amerikanischen Privatwirtschaft. Nicht weniger wichtig war der Transfer von Produktionsanlagen. Bis 1930 hatten ca. 80 amerikanische Firmen, an der Spitze die großen Automobilkonzerne, Zweigwerke in Deutschland errichtet. Amerikanische Kredite und amerikanisches Know how waren die Grundlage des labilen Gleichgewichts der Weimarer Republik in den Jahren 1924 bis 1929. 
Deutschland war durch den ersten Weltkrieg und die sich daran anschließenden Jahre der Revolution und bürgerkriegsähnlicher Zustände für nahezu ein Jahrzehnt von den wirtschaftlichen und technischen Entwicklungen auf dem Weltmarkt abgeschnitten. Erst jetzt wurde deutlich, in welchem Maße die Produktionsmet hoden in den USA während dieser Zeit erneuert worden waren. Seit ca. 1910 hatte sich dort eine neue wissenschaftliche Methode zur Rationalisierung von Arbeitsvorgängen durchgesetzt: der sogenannte Taylorismus, der $\mathrm{zu}$ einer erheblichen Steigerung der Arbeitsproduktivität beitrug. Wenn auch das Taylor-System bereits vor 1914 in Deutschland diskutiert und von vereinzelten Unternehmen übernommen worden war, so wurde es doch erst ab 1924 mit der Gründung des «Reichsausschusses für Arbeitsstudien» (REFA) zum anerkannten Vorbild der Betriebsorganisation im deutschen Kapitalismus. Es kam zu einer forcierten Industrialisierung von Wirtschaftszweigen, in denen noch die handwerkliche Fertigung vorherrschte (beispielsweise im Baubetrieb), sowie zu einer intensiven Rationalisierung bereits bestehender Industrien. Im großen Stil wurden amerikanische Produktionsmethoden und Lebensformen importiert. «Amerikanisiert» wurde seinerzeit so gut wie alles, vom Musikleben bis zur Filmproduktion, vom Bürobetrieb bis zum Zeitungs-Lay-out, vom bargeldlosen Zahlungsverkehr bis zum Drink. «Die Amerikanisierung des Abenteuers» überschrieb Richard Huelsenbeck einen satirischen Artikel in der «Literarischen Welt» (November 1926), in dem er den neuen Typus des Reisenden vorstellte, der fremde Kulturen im Rhythmus des laufenden Bandes zu erleben sucht. Diese Erscheinung der begeisterten Übernahme amerikanischer Erfindungen, Lebensart und Philosophie war die konkrete historische Grundlage des künstlerischen und literarischen «Amerikanismus»8.

10 Waren das Hochhaus, die Mechanisierung des Lebens, die Verwissenschaftlichung der Produktion und das Fließbandprinzip die äußeren Embleme dieser Weltanschauung, so bildete ihr Zentrum die Vorstellung, in den USA sei eine von Technikexperten verwaltete Gesellschaft entstanden, die in der Lage sei, die sozialen Probleme, die die europäischen Nationen zerrissen, sachlich-rational zu lösen.

11 In dem Industriellen Henry Ford verkörperten sich die Ideale, die die republikanische und die links-liberale deutsche Intelligenz in Amerika verwirklicht sah. Seine Autobiografie «Mein Leben und Werk» erschien 1923 in deutscher Übersetzung und wurde zu einem Bestseller der zwanziger Jahre ${ }^{9}$. Sie enthielt das Versprechen, «das wüste Feld der Industrie in einen blühenden Garten zu verwandeln» ${ }^{10}$. Kennzeichnend für Fords Betriebe waren die Rationalisierung der Planungs- und weitestgehende Mechanisierung der Produktionvorgänge. Als einer der ersten hatte er 1913 die Fließbandfertigung in die Automobilindustrie eingeführt. Fords Geschäftstheorie basierte auf der Ausweitung des Massenkonsums, bewirkt durch eine Senkung der Produktionskosten auf dem Wege der Automatisierung und Hebung der Kaufkraft durch relativ hohe Löhne.

Der SPD und den sozialdemokratisch orientierten Gewerkschaftsführern versprach Fords Devise: "Gutbezahlte Arbeiter beteiligen sich an keiner sinnlosen Umwälzung» ${ }^{11}$ die Beilegung der seit 1918 ständig gegenwärtigen Drohung der sozialen Revolution. In Fords Methoden entdeckte der einflußreiche bürgerliche Nationalökonom Friedrich von Gottl-Ottlilienfeld die Chance eines «weißen Sozialismus der reinen, tatfrohen Gesinnung» ${ }^{12}$. In seinem Buch "Fordismus. Über Industrie und technische Vernunft» aus dem Jahr 1924 preist er die Fordsche Politik als Beispiel dafür, daß die freie 
Entfaltung der im Kapitalismus schlummernden Kräfte die Klassenkonflikte lösen und die Harmonie aller Interessen sichern könne. Ford habe bewiesen, daß ein friedliches Zusammenleben von Kapital und Arbeit möglich sei, wenn nur die einander bekämpfenden Klassen sich als Partner begreifen und dem «arbeitsfrohen Dienst an der Gemeinschaft» unterordnen würden ${ }^{13}$.

Während Ford die gesundheitlichen Gefahren der äußersten Rationalisierung für die Arbeiter an seinen Montagebändern als fixe Ideen von «Salonexperten» und «berufsmäßigen Agitatoren» abtat ${ }^{14}$, bescheinigten ihm deutsche Nationalökonomen, Ingenieure und Schriftsteller, die als Touristen die Detroiter Werke besichtigt hatten, daß die Arbeiter in den Montagehallen nicht wie Proleten, sondern wie «Gentlemen» aussähen, die ihre monotonen Handgriffe «in sportlichem Gemütszustande» verrichteten.

So schildert sie der Reiseschriftsteller Heinrich Hauser, der seine Bücher seit 1928 mit eigenen Fotos illustrierte. Thema der 1931 erschienenen Reisereportage «Feldwege nach Chicago» ${ }^{15}$ ist die Unmenschlichkeit der Zivilisation und des Lebens in den großen Städten. Nur Detroit, die Fort-Stadt, begeistert den Autor. «Man arbeitet, aber es fließt kein Schweiß bei Ford», schreibt er über die Arbeitsbedingungen in Gießerei und Martins werk. Am Fließband beobachtet er: «Die Transportbänder bewegen sich oft mit überraschender Geschwindigkeit. Die Arbeiter laufen bei der Arbeit mit, oft rückwärts gewandt. Es entstand daraus ein fast sportlicher Eindruck, so etwas wie von der "Beinarbeit" eines Boxers». In die Schilderung der Mittagspause scheint sich die Erinnerung an einen Stadtbummel durch Paris zu mischen: «Glänzendes Essen in der Kantine: ein französischer Chef, "High-Priced". Seltsamer Geruch in der Fabrik: fast Duft - heißes Eisen riecht oft wie Blumen» ${ }^{16}$.

Solche Verhältnisse meinte Hauser an vereinzelten Stellen auch schon in der deutschen Industrielandschaft verwirklicht zu sehen. In dem ein Jahr vor seiner Amerikareise veröffentlichten Buch «Schwarzes Revier» preist er die Kruppschen Betriebe als «Humanisierung der Hüttenindustrie» ${ }^{17}$. Das Maschinenhaus erscheint ihm «wie eine Bühneninszenierung vom Bauhaus Dessau», die neuen Hochöfen «haben kaum mehr Ähnlichkeit mit dem, was man bisher unter Hochofenwerk oder Fabrik verstanden hat. Das ist vielmehr so, als wäre das Gerät eines chemischen Laboratoriums, gläserne Tische mit komplizierten Objektträgern, mit verschiedenartigsten Kolben und Reagenzgläsern, riesenhaft vergrößert in Zement, Stahl und Glas ausgeführt» ${ }^{18}$.

Im Krupp-Konzern begegnete Hauser der deutsche Kapitalismus auf seinem höchsten technischen Niveau: Präzisionsarbeit in gigantischen Dimensionen, technische Eleganz, Wissenschaftler im Management, Sauberkeit, durch Psychotechnik gespaltene und befriedete Arbeiterschaft. Dieser Fortschritt ist Hauser zufolge der neuen Generation der Unternehmer zu verdanken, die «vor allem von Amerika gelernt hat, daß eine Lösung des Arbeitsproblems auch dadurch möglicht ist, daß man den Arbeiter aus dem Fabrikationsprozeß so weit als möglich ausschaltet. Die große Rationalisierung gehört zu den Erfolgen dieser Erkenntnis» ${ }^{19}$.

17 Die Fotos, mit denen Hauser seine Reportage bebilderte, gleichen in Aufbau und Perspektive der gleichzeitigen professionellen Fotografie der Neuen Sachlichkeit. Auf ihnen dominieren Vertikale und Diagonale, Pathosformeln des Dynamischen, Aufstrebenden und Zukunftsweisenden der neuen industriellen Verfahren.

Um die Produktionskosten zu senken und auf dem Weltmarkt wettbewerbsfähiger zu werden, betrieben die deutschen Unternehmen in den zwanziger Jahren eine intensive 
Rationalisierung nach amerikanischem Vorbild. Durch die Übernahme der amerikanischen Produktionsmethoden glaubten Politiker, Gewerkschaftsführer und Intellektuelle des rechten wie des linken Lagers die Not der Nachkriegsjahre überwinden und bald den sagenumwobenen Lebensstandard der amerikanischen Gesellschaft erreichen zu können. In dieser Situation wurde Technik zur Zauberformel. «Die Technik hat die Tore des Paradieses gesprengt; durch den schmalen Eingang sind bisher nur wenige geschritten (die Dollarmillionäre), aber der Weg steht offen und durch Fleiß und Geist kann einst die ganze Menschheit jenen Glückskindern folgen», verkündete der Kulturphilosoph Graf CoudenhoveKalergi in seinem Buch «Praktischer Idealismus, Adel-Technikpazifismus» von $1925^{20}$. Als Alternative zum Schreckgespenst der sozialistischen Revolution propagierte er die «Weltrevolution der Technik». «Auch wir stehen vor einer Weltwende. Die Menschheit erwartet heute von der sozialistischen Ära den Anbruch des goldenen Zeitalters. Die erhoffte Weltwende wird, vielleicht, kommen: aber nicht durch Politik - sondern durch Technik; nicht durch einen Revolutionär, sondern durch den Erfinder $»^{21}$.

Die gleiche Auffassung vertrat der Bauhaustheoretiker Laszlo Moholy-Nagy in der Einleitung seines berühmten Buches «Malerei Fotografie Film» aus dem gleichen Jahr. «Der Techniker hat die Maschine in der Hand: Befriedigung momentaner Bedürfnisse. Aber im Grunde weit mehr: er ist Initiative der neuen sozialen Schichtung, Zukunfsebnender» ${ }^{22}$.

In einer Zeit, in der das deutsche Kultur- und Geistesleben von vehementen Richtungskämpfen geprägt war, schien eine eindeutige Zielstrebigkeit nur auf dem Gebiete der Technik erkennbar. "Wohin wir in Kunst und Literatur, in Musik und Dichtung streben», schrieb Leo Adler 1928 in der «Literarischen Welt», «wir wissen es im Grunde nicht, wissen kaum, was Kunst heute noch soll, kann und muß... Klar und vorerst unaufhaltsam ist nur der Siegeslauf der Technik» ${ }^{23}$.

Die Möglichkeit, eine Gesellschaft aufzubauen, die nach dem Modell technischer Effizienz funktioniert, schien sich am Beispiel Amerika bewahrheitet zu haben. Technik-Kult, Neue Sachlichkeit und «Amerikanismus» sind verschiedene Seiten des gleichen soziokulturellen Prozeßes: der forcierten Industrialisierung aller praktischen und geistigen Lebensverhältnisse. Bereits 1927 konnte man in der «BZ am Mittag» lesen, daß die Parole der Neuen Sachlichkeit ihr Zentrum letztlich in rein ökonomischen Erwägungen habe. «Vom modernen Büro, vom Fabrikbetrieb kam die Sachlichkeit her», heißt es dort. «Heute gibt es Gesellschaften, die bis ins raffinierteste Detail die Bürobetriebe durchdacht haben - natürlich die kaufmännischen zuerst - um schnelle Arbeit und sparsamsten Kraftverbrauch der Büroleute zu gwährleisten... Fabrik? Das Taylorsystem ist seit Jahrzehnten "neue Sachlichkeit". Ebenso die Psychotechnik» ${ }^{24}$. Berlin, so der Feuilletonist weiter, sei der Ursprungsort der neuen Parole: «Denn es ist noch unsentimentaler als Amerika, wenn es nur will».

Der Idee einer neuartigen Verbindung von ökonomischem Denken und künstlerischer Formgebung hatte sich bereits 1926 die Massenpresse angenommen. So erschien im März des Jahres in der Zeitschrift «UHU», dem populären Monatsmagazin des UllsteinVerlages, unter dem Titel «Die Schönheit der Technik. Die Geburt einer neuen Kunst» ${ }^{25}$ ein Artikel, der die Leser davon zu überzeugen suchte, daß die zunehmende Technisierung des Lebens nicht nur eine neue Stufe des wirtschaftlichen Fortschritts, sondern auch der Ästhetik darstelle, insofern die neuen Industriebauten, Maschinen und Produkte zu Formen gefunden hätten, die, dem Zweck, dem ökonomischen Kalkül 
und dem Material optimal entsprechend, nach einem neuen Schönheitssinn verlangten. Dessen naiven und reduzierten Grundsatz formuliert der Autor mit der Simplizität einer Bildlegende: «Sachlichkeit führt von selbst zu guten Formen», lautet der Kommentar zu einem Foto, das das Innere eines Desinfektionskessels abbildet ${ }^{26}$. Die bedeutendsten Künstler des Jahrhunderts sind dieser Überzeugung gemäß «die Schöpfer der modernen Technik», unsentimentale Erfinder, «unlyrisch bis ins Material: Stahl, Eisenbeton, Kautschuk». Illustriert ist der Aufsatz in der Hauptsache mit Fotografien von Sasha Stone, die die skulptur aie Eleganz einfacher technischer Formen am Beispiel eines Hochspannungsisolators, einer Mammutzange, eines Klavier hammers und eines Blitzableiters vor Augen führen sollen.

Im Geiste der Neuen Sachlichkeit ist ein Beitrag über die Rationalisierung des modernen Büros gehalten, der wenige Wochen später von demselben Magazin veröffentlicht und ebenso von Sasha Stone illustriert worden ist. Unter der Überschrift «Das loopferdige Büro - keine Utopie ${ }^{27}$ werden in ihm die Möglichkeiten zur Erhöhung der Arbeitsproduktivität durch Einsatz moderner Bürotechnik beschrieben.

Der bereits erwähnte Wirtschaftstheoretiker Friedrich Gottlottlilienfeld, der Wortführer der ideologischen Fordisierung in Deutschland, verkündete die Technik als den Motor, der die Entwicklung zu einer neuen humanen Gesellschaft antreibt. Um den Umschwung zu einer menschenwürdigen Gesellschaft in Gang zu bringen, «hätte nur Eine Art Diktatur einzugreifen: die Diktatur der Technischen Vernunft ${ }^{28}$.

Die «technische Vernunft» nicht nur als leitende Idee der Wirtschaft, sondern als das endlich gefundene Prinzip der Schöpfung, als «echte Religion» darzulegen, beabsichtigt Rudolf Schwarz mit dem Bildband «Wegweisung der Technik» von 1928 ${ }^{29}$. Eine Zusammenstellung von 14 Bildtafeln nach Fotografien von Albert Renger-Patzsch, die nichts anderes veranschaulicht, als daß in der Geschichte der Technik ähnliche Konstruktionsprinzipien vorkommen wie in der Welt der Bäume und der Pflanzen, weckt bei Schwarz mystische Gefühle. «Die Formwelt der Technik ist wie alle echten Formen tief und hoch, hinaufreichend bis zum Heiligen und hinab bis zu den Dämonen, zum einen berufen und zum anderen versucht, eine wirkliche Welt» ${ }^{30}$.

Die «Technik» als Göttin einer Ersatzreligion, die Träume von vollautomatisierten Betrieben, wie sie in der neusachlichen Literatur geschildert wurden, fanden ihr bildhaftes Pendant in den Fotografien der menschenleeren Maschinenparks und der von Arbeitern geräumten Fabrikationssäle, die Albert Renger-Patzsch für das Werkbund-Buch «Eisen und Stahl» (1931) geschaffen hat ${ }^{31}$. Auf den 97 abgebildeten Fotos verirrt sich nur selten ein Mensch auf das Produktionsgelände. Die Betrachtung der Bilder weckt den Eindruck, als seien die Schwungräder, Kräne und Fräsmaschinen Automaten, die eigenmächtig Stahlbleche, Kurbelwellen, Motorräder, Registrierkassen usw. hervorbringen, deren sich die Menschen dann nur noch zu bedienen hätten.

Im Zusammenhang mit dem erhöhten Interesse für industrielle Fertigungsmethoden waren Typisierung und Normung in den 20er Jahren zu Kernfragen der ästhetischen Auseinandersetzung geworden, in der Baukunst ebenso wie in der Typografie, im Bereich der Produktgestaltung ebenso wie auf dem Feld der Fotografie. Auch hier war es Albert Renger-Patzsch, der für die Erfahrung der typisierten Gegenstandswelt eine neue fotografische Form gefunden hat. Er begriff, daß das industrielle Produkt die ihm eigentümliche Ästhetik erst in der Steigerungsform der Wiederholung entfaltet, in dem Augenblick, da das serielle Prinzip als Grundzug jeder industriellen Fertigung im Bilde unmittelbar anschaulich wird. Der Rhythmus der standardisierten Dinge, die 
ornamentale Häufung des Immergleichen, gehört neben der Monumentalisierung von Maschinendetails zu den typischen Kennzeichen der Sachaufnahmen, die RengerPatzsch für seinen Bildband «Die Welt ist schön» zusammengestellt hat. Das im Spätherbst 1928 erschienene Buch darf wegen des großen Einflußes, den es auf die stilistische Entwicklung vieler junger Fotografen in Deutschland gehabt hat, als das Hauptwerk der neusachlichen Fotografie bezeichnet werden ${ }^{32}$.

Tauchen die Produzenten in der Fotografie der Neuen Sachlichkeit ausnahmsweise einmal auf, so geschieht das, um die Exaktheit, Übersichtlichkeit und Rationalität der neuen Produktionsverfahren zu veranschaulichen. Beispiele dafür sind die Fotos von Hein Gorny in dem Jubiläumsband, den die Pelikan-Werke Günther Wagner in Hannover 1938 zum hundertsten Jahrestag ihres Bestehens herausbrachten ${ }^{33}$. Dieser Bildband lehrt noch ein weiteres. Auch wenn die neusachliche Fotografie in erster Linie von der republikanisch eingestellten Intelligenz betrieben wurde, so ist sie doch keineswegs an die politische Herrschaftsform des Parlamentarismus gebunden. Da sie stärker in dem neuen Entfaltungsgrad der industriellen Erlebnisweise verankert war als in einem bestimmten politischen Klima, konnte sie mehr oder weniger ungebrochen in die Zeit der Nazi-Diktatur übergehen ${ }^{34}$. Die Prinzipien des Fordismus, die Heinrich Hauser so begeistert hatten, sehen wir in dem Pelikan-Bildband von Hein Gorny meisterhaft ins Bild gesetzt: wissenschaftliche Planung und Organisation der Produktion, Arbeiten ohne Schweiß, Massenfertigung, Typisierung, Präzisionsarbeit, saubere und gesunde Arbeitsplätze; Unternehmer, Manager, Meister und Arbeiter nicht «Gentlemen», sondern der Sprachregelung des Regimes entsprechend: «Kameraden» ${ }^{35}$.

Doch kehren wir zurück in die Jahre der Weimarer Republik. Unter den zahllosen Amerika-Büchern, die in den Jahren nach dem «Dawes»-Plan erschienen sind, waren Fotobildbände selten. Doch einer von ihnen machte Sensation: Erich Mendelsohns «AMERIKA. Bilderbuch eines Architekten», erschienen im Frühjahr $1926^{36}$.

Erich Mendelsohn war ein in Avantgardekreisen renommierter Architekt, als er im Oktober/November 1924 eine Studienreise durch die Vereinigten Staaten unternahm ${ }^{37}$. Vor allem der sogenannte Einsteinturm in Potsdam von 1920/21, Inbegriff der expressionistischen Architektur, hatte ihn bekannt gemacht. Finanziert hatte die Reise eines der größten deutschen Verlagshäuser: der Rudolf Mosse-Verlag in Berlin, im Austausch gegen eine Artikelserie für das «Berliner Tageblatt» oder ein Buchprojekt ${ }^{38}$. Mendelsohn, der sich bis dahin vor allem der Zeichnung bedient hatte, um visuelle Eindrücke festzuhalten, versuchte sich auf dem Feld der Fotografie und schuf ein Fotobuch mit 77 in Kupfertiefdruck reproduzierten Aufnahmen. Der Band, zwei Jahre später in einer erweiterten Fassung neu aufgelegt ${ }^{39}$, wurde zur maßgeblichen fotografischen Dokumentation Amerikas im Deutschland der Weimarer Republik. Die Fotos gaben denjenigen, die die Idee Amerika liebten, das Land aber nie mit eigenen Augen hatten sehen können, eine faszinierende optische Anschauung. «Amerika heute zu sehen», schreibt Mendelsohn in einem knappen Vorwort, «ist [...] ein perspektivischer Rausch. Erst hier erkennen wir die ganze Ungeheuerlichkeit der verneinenden Zivilisation, aber gleichzeitig in diesem Schwimmbrei schon die ersten Fixpunkte einer neuer Zeit.

Wer Amerika kennt, dem fällt es leichter, nun von der Spitze der Zeit zurückzurechnen, auf sich selbst zu schließen, anstatt ohne seine Kenntnis vom alten Nullpunkt aufzusteigen zum höchsten Stand. 

abschüttelt und nur «aus Zweck und Material den unserer Zeit entsprechenden Formenausdruck findet» ${ }^{45}$. Das ereignet sich jedoch weniger an den Schauseiten der Gebäude, jenen «Vorderfronten der Geldburgen», die noch ganz von den Zitaten historischer Machtzeichen leben, als an deren Rückseiten, die oft «jede unnötige Geste» vermeiden und «klar, unsinnlich, sachlich» gehalten sind ${ }^{46}$. Den vollkommensten Ausdruck «rationeller Architektur» erkennt Mendelsohn in den unrepräsentativen Industriebauten, allen voran den Getreidespeichern von Chicago, wo er «aus nackter Zweckform abstrakte Schönheit» entstehen sieht ${ }^{47}$. Zu der Aufnahme eines Hinterhofes 
in Detroit schreibt er: «Phantastische Wirkungen entstehen. Undurchbrochene Vertikale mit weißen leuchtenden Glasuren in die dunkle Gasse springend. Technische Schönheit, die neue Romantik» ${ }^{48}$.

Mit dieser Bemerkung hat Mendelsohn einen Wesenszug der Neuen Fotografie der zwanziger Jahre auf den Begriff gebracht, noch bevor diese sich in ihrem ganzen Spektrum entfaltet hatte. Fotografien technischer Schönheit als Bilder einer neuen industriellen Romantik hervorzubringen, war das Bestreben sowohl der Fotografen des Bauhauses als auch der Neuen Sachlichkeit.

\section{NOTES}

1. - Harry Graf Kessler, tagebuch eines Weltmannes, Literaturarchiv marbach am Neckar 1988, S. 341.

2. - Erich Mendelsohn, Rusland Europa Amerika. Ein architektonischer Querschnitt. Rudolf Mosse Buchverlag Berlin 1929.

3. - Ebenda, unpag. (S. 171).

4. - Ebenda, unpag. (S. 216).

5. - Ebenda, unpag. (S. 216).

6. - Ebenda, unpag. (S. 7).

7. - Ebenda, unpag. (S. 170).

8. - Siehe dazu die ausführiliche Studie von Helmut Lethen Neue Sachlichkeit 1924-1932. Studien zur Literatur des «Wiesßen Sozialismus», Stuttgart, 1970, Kapitel «Amerikanismus», S. 19-57.

9. - Im Paul List Verlag Leipzig.

10. - Ebenda, S. 314.

11. Henry Fort, Das größere Morgen, Paul List Verlag Leipzig 1926, S. 326.

12. - Friedrich von Gottl-Ottlilienfeld, Fordismus. Über Industrie und technische Vernunft, Jena 1924. Hier zitiert nach der 3. Erweiterten Auflge von 1926, S. 40.

13. - Ebenda, S. 38.

14. - Zit. Nach Lethen, a.a.O., S. 21.

15. - S. Fischer Verlag, Berlin.

16. - Ebenda, S. 230-233.

17. - S. Fischer Verlag, Berlin 1930, S. 44.

18. - Ebenda, S. 45.

19. - Ebenda, S. 97.

20. - Wien und Leipzig, S. 113.

21. - Ebenda, S. 105.

22. - Albert Langen Verlag, München, S. 30.

23. - Leo Adler. Kunst und Technik. In: Die Literarische Welt, Nr 10, 4. Jg., Berlin 9, März 1928, S. 77.

24. - Christian Buchholtz, Die neue Parole Sachlichkeit. In : BZ am Mittag, Berlin 13. April 1927.

25. - Berlin, 2. Jg., Heft 5, S. 54-62. Der Name des Autors, der mit dem Kürzel E.B. unterschreibt, ist nicht bekannt. 
26. - Dieselbe Auffasung vertritt ein jahr später Dr.Ing. Werner Lindner in dem Aufsatz "Der Ingenieur als Schöpfer neuer Formen. Architektur der Technik", in dem es heißt : "Reine Zweeckmäßigkeit ist immer schön”, In UHU, 3, Jg., Heft 7, Berlin, Apris 1927. Das Zitt auf S. 29.

27. - Von Fritz Zielesch, UHU Berlin, 2, Jg., Heft 7, April 1926, S. 52-59.

28. - In: Fordismus. Über Industrie und technische Vernunft. A.a.O., S. 39.

29. - Müller \& Kiepenheuer Verlag, Potsdam.

30. - Ebenda, S. 56.

31. - Verlag Hermann Reckendorf Berlin.

32. - Im Kurt Wolff Verlag, München.

33. - Während die Verwantwortlichen für Text, grafische gestaltung und Drucklegung des Buches genannt sind, wird der Name des Fotografen merkwürdigerweise an keiner Stelle erwähnt. Zur Autorschaft von Hein Gorny siehe Ausstellungskatalog "Hein Gorny", Fotogalerie Spectrum, Hannover 1972.

34. - Das kam insbesondere Albert Renger-Patzsch zugute, der in der zweiten Hälfte der dreißiger Jahre mit der Illustrierung mehrerer Firmenbildbände betraut wurde. Die bekanntesten Titel lauten: Vom Kraußschmied zur Kraußware. Zum fünfzigijähigen Bestehen der Kraußwerke, Privatdruck der Firma F.E. Krauß, o.O. und o.J. (Schwarzenberg, 1937); Kupferhammer Grünthal. Vierhundert Jahre deutscher Arbeitskultur. 1537-1937. Hrsg. Von der F.A. Lange Metallwerke AG Aue-Auerhammer/Kupferhammer grünthal, o.O. und o.J. (1937); Das siberne Erzgebirge. Bilder aus dem Erzgebirge. Mit einemen Vorwort von F.E. Krauß. Privatdruck der Kraußwerke Schwarzenberg 1940.

35. - in welchem Maße Taylorismus und Fordismus auch die Betriebsspolitik während des Nationalsozialismus bestimmt haben, hat Martin Rüther am Beispiel der Klöckner-HumboldtDeutz AG (KHD) eingehend dargelegt. In Zeitschrift für Unternehmensgeschichte, 33. Jg., Heft $2 / 1988$.

36. - Berlin, Rudolf Mosse Buchverlag. Von der Berliner Zeitschrift éDas Tagebuch“ nach den besten Bûchrn des Jahres 1926 gefragt, nannte Bertolt Brecht u.a. Mendelsohns "Amerika“: "Ausgezeichnete Fotos, die man eigentlich fast alle einzeln an die Wand heften kann...". In: bertolt Brecht, Gesammelte Werke, Bd. 18, Frankfurt am Main 1967, S. 51 and 52.

37. - Ein Großteil der Reise ist in dem Buch "Erich Mendelsohn. Briefe eines Zrchitekten", hg. Von Oskar beyer, München 1961; S. 59-76, dokumentiert. Ursprünglich hatte Meldelsohn beabsichtigt, auch nach Kalifornien zu reisen. Doch schon die Eindrücke in den Großstädten an der Ostküste erwiesen sich als so mannigfaltig und tief, daß er den Plan fallen ließ.

38. - Siehe Interview mit der Witwe Erich Meldelsohns, in: Ausstellungskatalog "The Drawings of Eric Meldelsohn". University Art Museum, University of California, Berkeley 1969, S. 37.

39. - Erich Mendelsohn, Amerika. Bilderbuch eines Architekten. Mit 100 meist eigenen Aufnahmen des Verfassers. Rudolf Mosse Buchverlag, Berlin 1928.

40. - Ebenda, S. IX.

41. - Einige der Aufnahmen stammten allerdings nicht von Meldelsohn, sonder, wie er in der Neuauflage von 1928 bekennen mußte, von dem detroiter Architekten K. Lönberg-Holm (a.a.O. S. é2é). Siehe dazu ausführlicher: Herbert Molderings, Mendelsohn, Amerika und der Amerikanismus. Nachwort des Reprints von Erich Mendelsohn. Amerika. Bilderbuch eines Architekten. Verlag Vieweg, Braunschweig 1991, S. 83-92.

42. - El Lissitzky, Das Auge des Architekten. Erich Meldelsohn. Amerika. Bilderbuch eines Architekten. Zitiert nach der Übersetzung in El Lissitzky, Proun und Xolkenbügel, Schriften, Brief, Dokumente, Dresden 1977, S. 64ff.
43. - A.a.O., S. 10
44. - A.a.O., S. 26.
45. - A.a.O., S. 63.
46. - A.a.O., S. 73. 
47. - A.a.O., S. 40.

48. - A.a.O., S. 42.

\section{RÉSUMÉS}

Neue Sachlichkeit in der Fotografie, das bedeutete Industrialisierung des Blicks, Technisierung des Empfindungsvermögens. Der neue Industrialisierungsschub ging von den USA aus. Im großen Stil wurden während der Weimarer Republik amerikanische Produktionsmethoden und Lebensformen importiert. Amerikanisiert wurde seinerzeit so gut wie alles, vom Musikleben bis zur Filmproduktion, von der Architektur bis zum Drink, vom Bürobetrieb bis zum ZeitungsLayout. Die begeisterte Übernahme amerikanischer Erfindungen, Lebensart und Philosophie war die Grundlage einer geistigen Strömung, die man «Amerikanismus» nannte. Der Vortrag zeigt am Beispiel von Fotografien Sasha Stones, Heinrich Hausers, Albert Renger-Patzschs, Hein Gornys und Erich Mendelsohns, daß der «Amerikanismus» zu den Kernbestandteilen der Neuen Sachlichkeit in der Fotografie gehört.

La « Nouvelle Objectivité » dans l'art photographique: cela signifiait l'industrialisation du regard, la contamination de la sensibilité par les finalités techniques. Le nouvel essor de l'industrialisation était parti des Etats-Unis. Sous la République de Weimar, on a procédé à l'importation en grand des méthodes de production et des styles de vie d'outre-Atlantique. Pratiquement tout fut "américanisé ", de la vie musicale à la production cinématographique, de l'architecture aux «drinks», du travail de bureau à la mise en page des journaux. A la base du courant qu'on a dénommé "américanisation», on trouve l'adoption enthousiaste des inventions américaines, de l'american way of life et de sa philosophie. Cette contribution fait apparaître, à partir de photos de Sasha Stone, de Heinrich Hauser, d'Albert Renger-Patzsch, de Hein Gorny et d'Erich Mendelssohn, que l'« américanisme » doit être considéré comme l'une des composantes fondamentales dans Fart photographique de la « Nouvelle Objectivité ». 